\title{
Accuracy of preoperative sampling diagnosis for predicting final pathology in patients with endometrial carcinoma: a review
}

\author{
David Lukanović $^{1,2}$, Miha Matjašič ${ }^{3}$, Borut Kobal ${ }^{1,4}$ \\ ${ }^{1}$ Department of Gynecology, Division of Gynecology and Obstetrics, Ljubljana University Medical Center, Ljubljana, Slovenia; ${ }^{2}$ Faculty of Medicine, \\ University of Ljubljana, Ljubljana, Slovenia; ${ }^{3}$ Center for Social Informatics, Faculty of Social Sciences, University of Ljubljana, Ljubljana, Slovenia; \\ ${ }^{4}$ Department of Gynecology and Obstetrics, Faculty of Medicine, University of Ljubljana, Ljubljana, Slovenia \\ Contributions: (I) Conception and design: All authors; (II) Administrative support: All authors; (III) Provision of study materials or patients: All \\ authors; (IV) Collection and assembly of data: All authors; (V) Data analysis and interpretation: All authors; (VI) Manuscript writing: All authors; (VII) \\ Final approval of manuscript: All authors. \\ Correspondence to: David Lukanović. Department of Gynecology, Division of Gynecology and Obstetrics, Ljubljana University Medical Center, \\ Zaloška 2, 1000 Ljubljana, Slovenia. Email: david.lukanovic@mf.uni-lj.si.
}

\begin{abstract}
Endometrial cancer (EC) is the most common gynecologic cancer. The most frequent symptom of this disease is postmenopausal bleeding. Diagnosis of EC must be histologically confirmed, and there are several methods for endometrial sampling to obtain cells or endometrial tissue. The first step in diagnosis should be ultrasound measurement of endometrial thickness, followed by endometrial sampling, which can be performed by office endometrial biopsy, hysteroscopic biopsy, or dilatation and curettage (D\&C). The review in this article was carried out to present previously published studies, comprehensively evaluate method performance (i.e., overall accuracy of preoperative sampling in patients with endometrial carcinoma, and overall agreement on grade and histological subtype between preoperative endometrial sampling and final diagnosis), and determine which sampling method is most accurate on the basis of the statistical data in the studies analyzed. From the literature analyzed and examined, it can be concluded that preoperative endometrial sampling is not always the best predictor of final histology in EC and has its limitations. In surgical decisions based only on preoperative sampling, a biopsy should be made with caution, and it is necessary to take other parameters into account. Inadequate grading leads to suboptimal clinical management, mainly in early-stage tumors. This review showed that, although hysteroscopic biopsy was mainly associated with the highest tumor grade agreement, and although D\&C showed the highest overall accuracy in detecting endometrial carcinoma, the data do not therefore reliably indicate which method yields the most precise results. The results of this review indicate that further studies on larger samples and with greater statistical power are needed to accurately define the role and type of preoperative sampling methods.
\end{abstract}

Keywords: Endometrial cancer (EC); biopsy; endometrial sampling; accuracy; sample size

Submitted May 31, 2020. Accepted for publication Jul 31, 2020.

doi: $10.21037 /$ tcr-20-2228

View this article at: http://dx.doi.org/10.21037/tcr-20-2228

\section{Introduction}

Endometrial cancer (EC) is the most common gynecological malignancy in high-income countries (1). Eighty percent of all cases are discovered in an early phase (stage I or II) because the disease reveals itself fairly early in its course through irregular vaginal bleeding. It results in a good prognosis, with a 5-year overall survival (OS) rate of $90 \%$ to $95 \%(2,3)$. In most cases, endometrial carcinoma is diagnosed with a preoperative sampling method even before hysterectomy is performed. Endometrial samples can be obtained on an outpatient basis (biopsy or outpatient hysteroscopy) or through a procedure under short-term general anesthesia [surgical hysteroscopy, or dilatation 
and curettage (D\&C)] (4-6). The oldest method for taking samples is D\&C. It is regarded as an outdated diagnostic procedure because more modern, less invasive tests are available (5). There is a trend toward minimally invasive exams using endometrial biopsy, vaginal ultrasound scan, and hysteroscopy (HSC) $(5,7)$. Endometrial biopsy with an aspirational catheter is an outpatient procedure that may have less diagnostic value because only tiny fragments of tissue are removed and therefore a significant number of missed lesions are to be expected. A more recent diagnostic method for uterine mucosa sampling is hysteroscopic biopsy, which is now considered the gold standard in uterine cancer diagnostics (8). The method is also more sensitive for detecting benign pathologies such as endometrial polyps or submucosal myoma $(5,7)$. However, the European Society for Medical Oncology (ESMO), European Society for Radiotherapy \& Oncology (ESTRO), and European Society of Gynaecological Oncology (ESGO) consensus conference on EC [2016] suggests that the optimal method for obtaining histological characteristics is $\mathrm{D} \& \mathrm{C}$ because it is superior to pipelle biopsy in terms of accuracy of the tumor grade $(4,9,10)$. On the other hand, the latest ESMO Clinical Practice Guidelines point out that the question of what the best diagnostic strategy is in patients with vaginal bleeding still remains controversial (7). As Martinelli et al. (11) stress in their article, obtaining an endometrial/ uterine sample is of utmost importance; however, the accuracy of these evaluations is not well understood.

The first step in diagnosis should be ultrasound measurement of endometrial thickness, using a cutoff point of 3 or $4 \mathrm{~mm}$, followed by endometrial sampling $(1,7)$. When planning the extent of EC surgery, it is necessary to be aware that the histology results of the preoperative biopsy and the final sample may differ in up to $15 \%$ to $25 \%$ of cases $(12-17)$, or even $32 \%$ to $97 \%$ of cases according to some sources (18-20). From this perspective, the histology and grade EC are one of the most important predicting factors of disease outcome and of the likelihood of nodal metastasis. Moreover, preoperative tumor grade is an important cornerstone for determining the extent of surgical treatment (18). Discrepancy of the tumor grade may lead to either insufficient treatment due to downgrading (underestimating the risk of regional lymph node spread) on the one hand, or upgrading (overestimating risk) on the other hand, leading to overly extensive surgery that unnecessarily exposes the patient to the complications of surgery and its aftereffects $(5,6,18)$.

Therefore the review in this article was carried out in order to present published studies, to comprehensively evaluate the method performance (i.e., overall accuracy of preoperative sampling in patients with endometrial carcinoma, overall agreement on grade and histological subtype between preoperative endometrial sampling and final diagnosis), and to determine which sampling method is most accurate on the basis of the statistical data in the studies analyzed.

\section{Methods}

The process of selection of articles is described in Figure 1. A systematic literature search was conducted using Medline, Cochrane, and ClinicalTrials. All known synonyms were used for the following keywords: endometrial sampling and biopsy.

The analysis included all clinical studies (retrospective or prospective) describing the evaluation of the diagnostic accuracy of endometrial sampling in women with endometrial carcinoma. In eligible studies, the index test included office endometrial biopsy, hysteroscopy, hysteroscopic biopsy, or dilatation and curettage $(\mathrm{D} \& \mathrm{C})$; the reference standard had to be hysterectomy. The outcome measure had to be tumor grade, histological subtype, or both.

All research articles in English published between 2010 and 2020 were reviewed (Figure 1). The article examines studies that contain the latest clinical practices for treating endometrial carcinoma in the past 10 years. It should also be noted that the focus of this article is only on research articles. Conference presentations and reports were excluded because the goal was to focus on the most carefully evaluated material. Potentially relevant research articles were identified by examining the abstracts or the articles as a whole.

\section{Results}

Sixty-six articles were identified and screened at the title and abstract levels. Forty-three articles were excluded for any of the following reasons: because they reported the evaluation of the diagnostic accuracy of endometrial sampling in women with postmenopausal bleeding, because there was absence of hysterectomy or preoperative histology, because the percentage of agreement was lacking, or because there were no possibilities for calculating these from the data available. Four of them were meta-analyses. Thus 19 articles, summarized in Table 1, represented the object of 

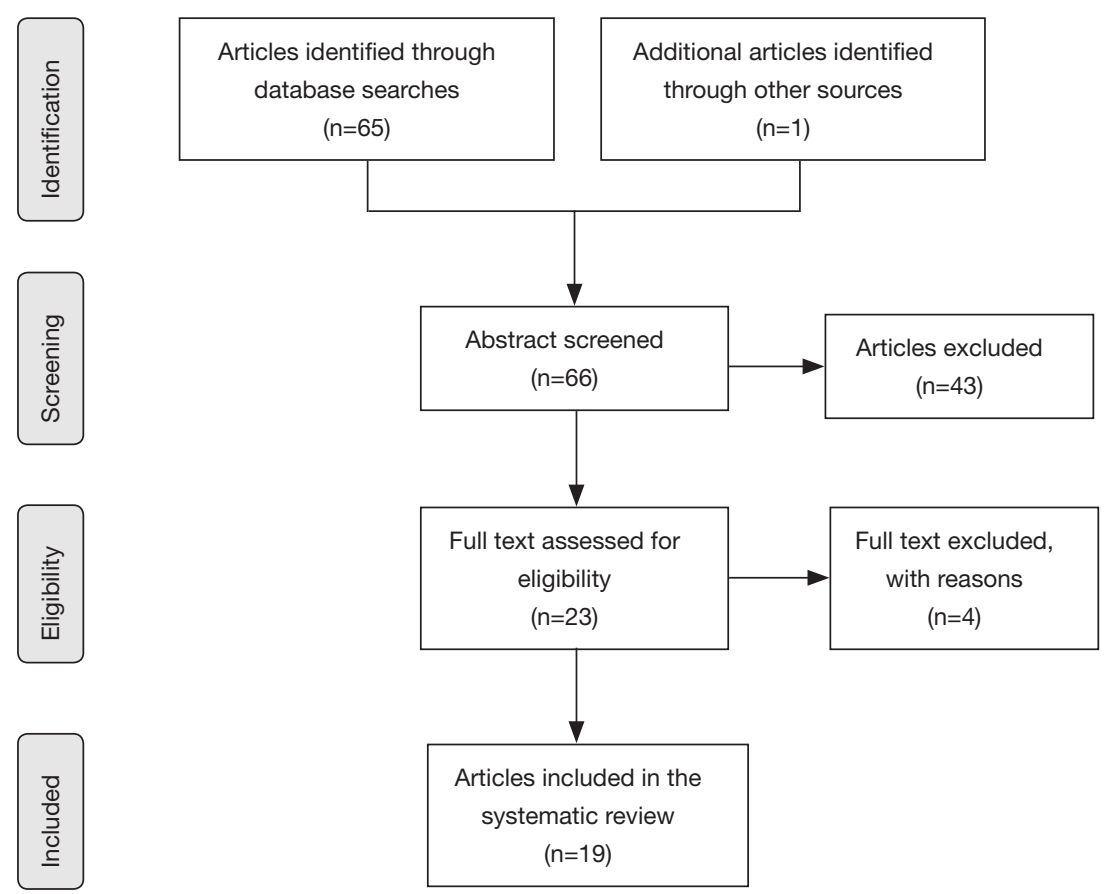

Figure 1 Flowchart of article selection process.

this review. Most of studies are retrospective; only the study by Ørtoft et al. (19) is prospective.

Nine articles were identified in which the authors used $\mathrm{D} \& \mathrm{C}$, eight in which the authors used hysteroscopic biopsy, five in which the authors used office endometrial biopsy, one in which the author used all three methods, and three in which the authors used D\&C and office endometrial biopsy. Hysteroscopic biopsy can be done either under general anesthesia or in an office setting, but most of studies in Table 1 did not report the way hysteroscopic biopsy was performed therefore we analyzed them together. Table 1 shows that the authors that used multiple methods in assessing the accuracy of preoperative sampling did not provide information regarding their methods and the results for each method separately, but for all the methods together. Therefore, it was not possible to obtain and assess the method performance information (e.g., the overall accuracy, the overall grade agreement, etc.).

Regarding the methods performance, Table 1 also shows that the values of sample sizes, overall accuracy, and overall agreement varied greatly among the studies and methods. Thus, in order to describe a set of observed data accurately, measures of central tendency were calculated and reported (i.e., mean and median) as well as the standard deviation. Furthermore, because the mean approach is highly sensitive to large values (i.e., skewed distribution), large values (e.g., sample size, value of agreement, and accuracy) will have an enormous impact, making the mean larger than the actual distribution of data would suggest. Therefore, the Table 1 also shows the median value, which is more robust and thus better suited for skewed distributions.

Regarding the sample size, it can be observed (Table 1) that the largest sample size was in studies that analyzed data with the $\mathrm{D} \& \mathrm{C}$ (a median value of 134 and a mean value of 159), followed by studies that analyzed data with the hysteroscopic biopsy (a median value of 75.5 and a mean value of 95), whereas the office endometrial biopsy had a median value of 102 (mean value 88.6). Moreover, in four studies the sample size was only reported for all methods altogether, and therefore it was not possible to calculate central tendency measures for each method separately. Based on the results obtained, it seems that there is a problem of generalizability of the results obtained from these studies due to the large differences between study sample sizes. Here should be noted that sample sizes should not be either too large or too small because both have limitations that can compromise the conclusions drawn from the studies. Too small a sample size may prevent the findings from being extrapolated, whereas too large a sample size may amplify the detection of differences, 
Table 1 Review of articles according to sample size, overall accuracy, observed agreement, and downgrading and upgrading based on D\&C, hysteroscopic biopsy, and office endometrial biopsy

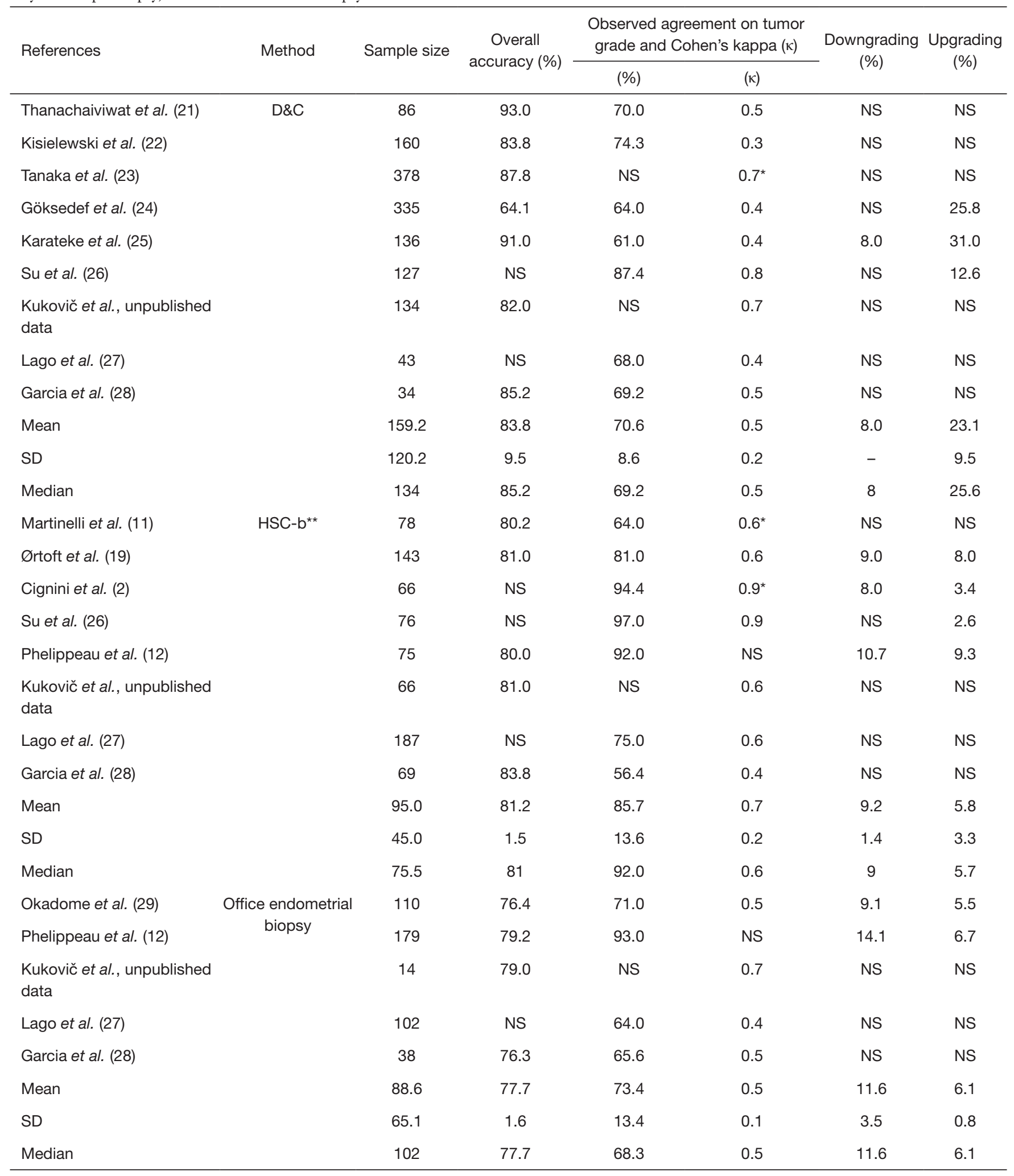

Table 1 (continued) 
Table 1 (continued)

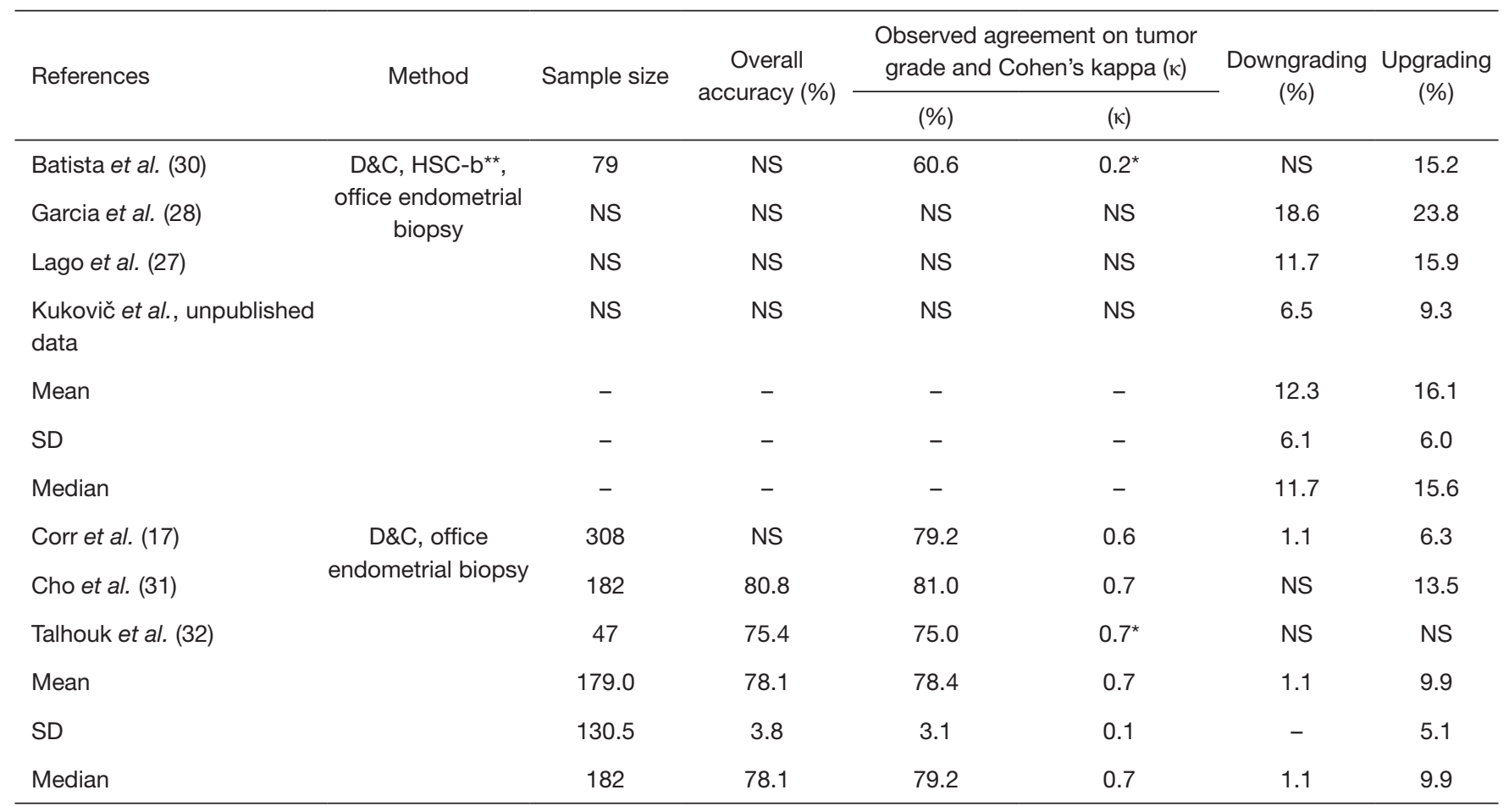

*, articles that also reported the P value; ${ }^{* *}$, hysteroscopic biopsy. NS, not stated.

emphasizing statistical differences that are not clinically relevant (33). Therefore, some of the results obtained from these studies should be interpreted with caution.

Regarding the methods, overall accuracy for predicting endometrial carcinoma, overall agreement on tumor grade, and Cohen's kappa, it can be observed that each of these methods differ, but on average all the methods analyzed had high overall accuracy and high overall agreement on tumor grade (Table 1). It can also be observed that, based on the value of the median, overall accuracy was the highest for the $\mathrm{D} \& \mathrm{C}$ (a median value of $85.2 \%$ ), whereas overall agreement was the highest for the hysteroscopic biopsy (a median value of $92 \%$ ). Furthermore, in terms of Cohen's kappa it can be observed that the $\mathrm{D} \& \mathrm{C}$ and office endometrial biopsy showed "moderate" agreement (mean $\kappa$ value of 0.5 , respectively) on tumor grade, whereas the hysteroscopic biopsy showed "good" agreement (mean $\kappa$ value of 0.7 ) on tumor grade. Here should be noted that the kappa result could be interpreted as follows: values $<0.0$ as indicating no agreement, $0.01-0.20$ as none to slight, $0.21-0.40$ as fair, $0.41-0.60$ as moderate, $0.61-0.80$ as substantial, and $0.81-$ 1.00 as almost perfect agreement (34). Moreover, only five studies also reported the $\mathrm{P}$ value of Cohen's kappa. When interpreting Cohen's kappa, it is important to bear in mind that the estimated Cohen's kappa itself could be due to chance, which means that the results obtained are probably not true. Therefore, it is important to calculate and report a $\mathrm{P}$ value, which tests whether the estimated Cohen's kappa is not due to chance.

Regarding "upgrading" and "downgrading", for the overview only data were used that were reported in the studies. Studies that did not report these data were labeled as "not stated" (Table 1). As Table 1 shows, upgrading was reported in only three studies for the D\&C, with a median value of $25.6 \%$. Four studies analyzing the hysteroscopic biopsy reported upgrading with a median value of $5.7 \%$, and upgrading for office endometrial biopsy was $6.1 \%$. In studies that provided information for all methods together, upgrading had a median value of $15.6 \%$. In studies that analyzed only D\&C and office endometrial biopsy together, upgrading had a median value of $9.9 \%$.

\section{Discussion}

From the literature analyzed and examined, it can be concluded that preoperative endometrial sampling is 
not always the best predictor of final histology in EC and has its limitations. In surgical decisions based only on preoperative sampling, a biopsy should be made with caution, and it is necessary to take other parameters into account. Our review showed that, although hysteroscopic biopsy was mainly associated with the highest tumor grade agreement, and although D\&C showed the highest overall accuracy in detecting endometrial carcinoma (see Table 1), the data therefore do not reliably indicate which method yields the most precise results. However, it would be also interesting to accurately analyze difference between office hysteroscopic biopsy and hysteroscopic biopsy under shortterm general anesthesia.

Furthermore, in the articles analyzed the authors did not report whether they also calculated the sample size needed to produce a confidence interval estimate with a specified margin of error (precision). If the sample size calculation is not done at all, it will affect the power (i.e., calculations that show the number of patients required in order to avoid a type I or type II error) and value of a study, and thus influence the possibility that the results obtained may be incorrect (35). Moreover, only one study was found by Cignini et al. (2), in which they reported that they did not perform any power analysis to determine the sample size and also strongly solicited further studies on larger cohorts and with greater statistical power. Such a study could accurately define the role and type of preoperative sampling methods. When data in this review (Table 1) were compared with meta-analyses by Van Hanegem et al. (14), who analyzed the accuracy of endometrial sampling in women with postmenopausal bleeding, it was found that most studies suggest that more research on this subject using larger samples is needed, given the prevalence of EC and atypical hyperplasia $(2,11,12,14,25,26,29)$. All of this could indicate that there is a need for more studies that will also account for power and sample size when comparing the performance of various methods.

According to consensus among ESMO, ESTRO, and ESGO (4), it is important for all patients with a risk of cancer, particularly patients with postmenopausal bleeding and a hyperplastic endometrium at ultrasound, to be investigated with a preoperative biopsy (only endometrial biopsy or $\mathrm{D} \& \mathrm{C}$ are mentioned) in order to avoid uterine morcellation, which poses a risk of spreading unsuspected cancerous tissue, notably endometrial carcinomas or sarcomas, beyond the uterus and may make the pathological assessment of myometrial invasion extremely difficult. Furthermore, a preoperative biopsy prevents the discovery of an unexpected malignancy after inadequate surgery (subtotal hysterectomy and/or preservation of the ovaries in a postmenopausal patient, with incomplete staging) (4). Looking at non-endometrioid uterine pathology, its diagnosis could be even more challenging than the endometrioid counterpart, but it is of utmost importance. Treatment of these rare tumors is more complex and requires expertise in gynecologic oncology (11). In addition, measuring relative telomere length (RTL) in circulating serum cfDNA may be a potentially valuable, noninvasive, simple, and relatively inexpensive diagnostic tool for early detection of EC. The high performance of cfDNA RTL even in earlystage EC could be useful for allowing early and prompt treatment and avoiding disease progression. However, its diagnostic performance seems unsatisfactory for detecting cancer progression, grading, and staging (36). All of the various data presented in the studies should be taken into consideration to establish the surgical plan and preoperative information for patients with early-stage endometrioid EC. Moreover, the need for caution should be emphasized in planning patient management based on the initial histological findings (21). Inadequate grading leads to suboptimal clinical management, mainly in early-stage tumors. Recommendations from the ESMO, ESTRO, and ESGO suggest a mandatory work-up, which must include family history, a general assessment and inventory of comorbidities, geriatric assessment (if appropriate), clinical examination, including pelvic examination, transvaginal or transrectal ultrasound, and a complete pathology assessment (histotype and grade) of an endometrial biopsy or curettage specimen (4). A history of breast cancer is a risk factor for the development of endometrial pathologies. However, women undergoing tamoxifen therapy after breast cancer surgery are not at increased risk of EC compared with women undergoing third-generation nonsteroidal aromatase inhibitor therapy or no treatment. Transvaginal ultrasound has a low concordance level with hysteroscopic findings, but to date there is no consensus on the best method of screening asymptomatic (without vaginal bleeding) tamoxifen users to select patients who should be addressed with HSC and targeted endometrial biopsy (37).

There were difficulties in analyzing "upgrading" and "downgrading" because some studies [Kukovič, 2019, unpublished data, $(27,28,30)]$ did not provide data regarding each method separately but for all methods together. On the other hand, some of these studies did provide some results for each method separately (overall accuracy, 
observed agreement on tumor grade, and Cohen's kappa). Considering that only three studies (24-26) on D\&C reported on "upgrading", this cannot be generalized for the general population (mean value $23.1 \%$ with SD 9.5). Moreover, some studies [Kukovič, 2019, unpublished data, $(27,28,30)]$ reported upgrading and downgrading for all three methods together regardless of the manner in which the sample was derived (median value for upgrading $15.6 \%)$. Two studies $(17,31)$ reported on upgrading for two methods together (median value for upgrading 9.9\%). Only these last results are comparable with results from meta-analyses performed by Visser et al. (18), who reported clinically relevant upgrading in $8.5 \%$ of cases, regardless of the method.

The vast majority of shifts presented in the literature occur from low (G1) to intermediate (G2) grade; only a small minority $(0.5-5 \%$ in various studies) of G1 tumors are reclassified as high grade based on final surgical pathology (38). A meta-analysis by Visser et al. (18) points out that one of the main reasons for disagreement on grade between endometrial sampling and final diagnosis could be the limited amount of tissue often obtained by preoperative endometrial sampling, which can lead to difficulties in assessing tumor grade (18). Grade shifts on final pathological assessments may be partly explained by the volume of tissue available for examination. A larger volume of tissue may allow better assessment of the solid growth component and avoidance of misleading sampling issues in mixed endometrioid/non-endometrioid tumors (38). Leitao et al. (10) showed that grade shifts are less frequent when preoperative sampling is done using $\mathrm{D} \& \mathrm{C}$ as compared to office aspiration biopsy. However, in this review and in meta-analyses by Visser et al. (18), hysteroscopic biopsies show a higher overall agreement on tumor grade compared with $\mathrm{D} \& \mathrm{C}$, suggesting that not only the amount of tissue, but also the accuracy of sampling is important. D\&C remains the most extended method at the initial workup of abnormal bleeding, as can also be seen from the size of samples shown in Table 1. A recent study by Di Spiezio Sardo et al. (39) confirmed that hysteroscopic guided "grasp" endometrial biopsy provides a more accurate diagnosis of EC histology type and tumor grade compared to blind endometrial biopsy obtained using the Novak curette. Di Spiezio Sardo et al. (39) also described the limitations of their study, which are a small sample size and the fact that the study design was retrospective.

Studies analyzing hysteroscopic biopsy showed that hysteroscopic biopsy has the highest observed agreement on tumor grade and also the highest value of Cohen's kappa. In agreement with most studies in our review, further studies on a larger sample are warranted for evaluating hysteroscopic biopsies (11). Lago et al. (27) also point out that neither the accuracy of hysteroscopic biopsies nor the accuracy of the tumor grade between different diagnostic methods has been accurately compared in the literature yet, and therefore no preference in the preoperative diagnostic method can be established.

An incorrect preoperative histological diagnosis can lead to unsatisfactory primary surgical treatment and, with underestimation of the disease, can lead to avoidance of lymphadenectomy in cases of high-risk disease (18). Even though the main guide for the type and extent of surgical treatment is the histological result of a biopsy, expert imaging examinations are urgently needed and of great importance because they can be used to assess tumor size and growth into the myometrium, cervix, and surrounding tissues, and to assess the status of the regional lymph nodes.

A study by Maneschi et al. (40) argued that preoperative tumor risk assessment was a strong predictor of risk. The issue of shifts between preoperative and final surgical pathology interpretation only becomes meaningful if preoperative histological features are used to guide surgical staging decisions. In spite of the frequent shift from a lowrisk to a high-risk classification based on final pathology, avoiding lymphadenectomy in low-grade EC has been shown to have no deleterious impact on overall or diseasefree survival (41-43). Mariani et al. (44) state that, following the protocol from the Mayo Clinic, only $1 \%$ of cases with positive lymph nodes would be missed if more than $40 \%$ of patients with low- and medium-risk disease did not undergo lymphadenectomy. A compromise between complete lymphadenectomy and complete avoidance of it is a biopsy of the sentinel lymph node with ultrastaging, which successfully bridges the gap between $15 \%$ and $25 \%$ of incorrect histological results of preoperative biopsy and detection of lymph node metastases even in low- and medium-risk patients $(3,4,9)$. Using the Mayo Clinic protocol yields important prognostic information for helping decide on adjuvant treatment in the case of a prognostically worse type of cancer after receiving a final histological result. Notwithstanding all of this, Helpman et al. (38) point out that decisions regarding a universal versus selective surgical staging policy—and setting an acceptable cutoff for selective staging-will undoubtedly vary between institutions and surgeons. On the one hand, we also have to take into account that gynecological 
cancer management in older people remains a current challenge. It is important to overcome the common habit of undertreating elderly patients because they are more fragile and have a lower life expectancy than their younger counterparts. Standard EC surgical management notably includes pelvic and/or lumbo-aortic nodal staging (45). Only Vaknin et al. $(46,47)$ found that a significantly higher number of lymph nodes were removed in patients $<70$ years. Otherwise, the literature comparing lymphadenectomy performed via laparoscopy, robotic surgery, or laparotomy in elderly women with EC did not report any significant difference. Therefore, minimally invasive surgery in elderly women with EC also seems a valid option for lymphadenectomy (45). On the other hand, gynecological cancer treatment significantly affects the fertility of women of reproductive age $(48,49)$. When a patient is to make an informed decision about her preferred treatment choice, it is important to include the patient's oncologist and gynecologist, a reproductive medicine specialist, a clinical psychologist, and if possible a medical ethics expert in the process $(50)$. In order to improve the quality of life and the survival of patients, improving fertility preservation techniques has become an important research topic $(51,52)$. The differentiation of EC is the most important predictor of stage. Duska et al. (53) reviewed women younger than 40 with EC and showed that only G1 EC could predict stage 1 disease among them. Therefore, endometrial biopsy has been the cornerstone examination for the diagnosis of EC. Due to the very difficult grading diagnosis in type I EC, all specimens should be examined by two pathologists: in the case of a positive diagnosis, a second evaluation is mandatory. We should also take into consideration that fertility-sparing surgery cannot be recommended for patients with stage IA, type I, and G3 because the absolute prognosis is worse compared to a patient undergoing radical surgery (54).

It should be mentioned that endometrial cytology has recently been reported as a useful diagnostic method with high sensitivity and specificity in detecting endometrial malignancies. Okadome et al. (29) was the first to reported that endometrial cytology had a higher evaluable rate for EC types and that endometrial cytology may complement endometrial biopsy in preoperative women with EC. A meta-analysis by Wang et al. (55) evaluated the diagnostic accuracy of endometrial cytology for EC compared with histological diagnosis. A meta-analysis showed that endometrial cytology had high diagnostic accuracy and could serve as a test to confirm or exclude endometrial atypical hyperplasia or cancer. In addition, Wang et al. (55) point out that endometrial cytology is inexpensive, is tolerated well, and can be performed without anesthesia in an outpatient clinic. It is now the most common test for an initial evaluation of EC in Japan (29,55), and it has been encouraged as a first-level screening method for women at high risk for EC (55-57). However, Okadome et al. (29) pointed out in their conclusion that both endometrial biopsy and cytology have advantages in prediction, and that this merits further investigation. On the other hand, Casarin et al. (58) investigated the correlation between glandular cells detected at a preoperative cervical smear and the histological findings and oncologic outcomes in patients undergoing surgery for EC. The conclusion was that cervical stromal invasion and the presence of glandular cells at the preoperative cervical smear might predict the occurrence of local recurrence in EC (58).

An interesting study by Yi et al. (8) analyzed the costeffectiveness of biopsy strategies for EC diagnosis in women with postmenopausal bleeding. The study compared pipelle sampling curette versus D\&C. The base case analysis suggested that pipelle was not only equally effective but also less costly based on Medicare reimbursement when compared to D\&C. In one-way sensitivity analyses and Monte Carlo probabilistic sensitivity analysis, pipelle remained the more cost-effective sampling strategy even after accounting for the sampling failure rate associated with each sampling strategy. Yi et al. (8) concluded that pipelle is the more cost-effective sampling strategy compared to D\&C for EC diagnosis. From the costeffectiveness perspective, the higher sampling failure rate of pipelle should not be regarded as a limitation in its clinical application. However, in their analyses the authors suggest that future studies should focus on in-office diagnostic HSC, which, unlike in the US, is already widely used in Europe for diagnosis of EC (8).

\section{Conclusions}

The optimal method for preoperative evaluation of endometrial carcinoma is not yet defined; it still remains a challenge and varies between institutions and surgeons. There is still no clear consensus in the literature regarding the preoperative sampling method with the highest accuracy. Nevertheless, this review showed that hysteroscopic biopsy had the highest observed agreement on tumor grade and 
the highest Cohen's kappa. Based on the studies evaluated and their conclusions, preoperative endometrial sampling was found to be only a modest predictor of postoperative histological grading.

The results of this review indicate that further studies should be designed to include a sufficient number of patients to adequately address the research question because sample size calculations are a key component of clinical research. The emphasis in most of these studies is on finding the magnitude of difference between methods. Such a study could accurately define the role and type of preoperative sampling methods.

\section{Acknowledgments}

The authors thank Miha Brvar for technical assistance and Donald Reindl for assistance with the English translation of the text.

Funding: None.

\section{Footnote}

Provenance and Peer Review: This article was commissioned by the Guest Editor (Antonio Simone Laganà) for the series "Endometrial Cancer" published in Translational Cancer Research. The article was sent for external peer review organized by the Guest Editor and the editorial office.

Conflicts of Interest: All authors have completed the ICMJE uniform disclosure form (available at http://dx.doi. org/10.21037/tcr-20-2228). The series "Endometrial Cancer" was commissioned by the editorial office without any funding or sponsorship. The authors have no other conflicts of interest to declare.

Ethical Statement: The authors are accountable for all aspects of the work in ensuring that questions related to the accuracy or integrity of any part of the work are appropriately investigated and resolved.

Open Access Statement: This is an Open Access article distributed in accordance with the Creative Commons Attribution-NonCommercial-NoDerivs 4.0 International License (CC BY-NC-ND 4.0), which permits the noncommercial replication and distribution of the article with the strict proviso that no changes or edits are made and the original work is properly cited (including links to both the formal publication through the relevant DOI and the license). See: https://creativecommons.org/licenses/by-nc-nd/4.0/.

\section{References}

1. Amant F, Raza MM, Koskas M, et al. Cancer of the corpus uteri. Int J Gynaecol Obstet 2018;143:37-50.

2. Cignini, $\mathrm{P}$, Vitale, SG, Laganà AS, et al. Preoperative work-up for definition of lymph node risk involvement in early stage endometrial cancer: 5 -year follow-up. Updates Surg 2017;69:75-82.

3. Franchi M, Garzon S, Zorzato PC, et al. PET-CT scan in the preoperative workup of early stage intermediate- and high-risk endometrial cancer. Minim Invasive Ther Allied Technol 2020;29:232-9.

4. Colombo N, Creutzberg, C, Amant F, et al. ESMOESGO-ESTRO Consensus Conference on Endometrial Cancer: diagnosis, treatment and follow-up. Ann Oncol 2016;27:16-41.

5. Šegedin B, Merlo S, Smrkolj Š, et al. Priporočila za obravnavo bolnic z rakom materničnega telesa. 2018. Available online: https://www.onko-i.si/fileadmin/onko/ datoteke/Revija_Onkologija/Priporocila_za_obravnavo_ bolnic_z_rakom_maternicnega_telesa.pdf

6. NCCN clinical practice guidelines in oncology. Uterine neoplasms, Version 1.2018. 2018. Available online: http:// www.nccn.org/professionals/physician_gls/pdf/uterine.pdf

7. Colombo N, Preti E, Landoni F, et al. Endometrial cancer: ESMO clinical practice guidelines for diagnosis, treatment and follow-up. Ann Oncol 2013;24:vi33-8.

8. Yi Y, Bryce CL, Adambekov S, et al. Cost-effectiveness analysis of biopsy strategies for endometrial cancer diagnosis in women with postmenopausal bleeding: pipelle sampling curette versus dilatation \& curettage. Gynecol Oncol 2018;150:112-8.

9. Rodolakis A, Biliatis I, Morice P, et al. European Society of Gynecological Oncology Task Force for Fertility Preservation: clinical recommendations for fertilitysparing management in young endometrial cancer patients. Int J Gynecol Cancer 2015;25:1258-65.

10. Leitao MM, Jr Kehoe S, Barakat RR, et al. Comparison of $\mathrm{D} \& \mathrm{C}$ and office endometrial biopsy accuracy in patients with FIGO grade 1 endometrial adenocarcinoma. Gynecol Oncol 2009;113:105-8.

11. Martinelli F, Ditto A, Bogani G, et al. Accuracy of preoperative hysteroscopic guided biopsy for predicting final pathology in uterine malignancies. J Cancer Res Clin 
Oncol. 2017;143:1275-9

12. Phelippeau J, Canlorbe G, Bendifallah S, et al. Preoperative diagnosis of tumor grade and type in endometrial cancer by pipelle sampling and hysteroscopy: results of a French study. Surg Oncol 2016;25:370-7.

13. Leitao MM Jr, Kehoe S, Barakat RR, et al. Accuracy of preoperative endometrial sampling diagnosis of FIGO grade I endometrial adenocarcinoma. Gynecol Oncol 2008;111:244-8.

14. Van Hanegem N, Prins MMC, Bongers MY, et al. The accuracy of endometrial sampling in women with postmenopausal bleeding: a systematic review and meta-analysis. Eur J Obstet Gynecol Reprod Biol 2016;197:147-55.

15. Larson DM, Johnson KK, Broste SK, et al. Comparison of $\mathrm{D} \& \mathrm{C}$ and office endometrial biopsy in predicting final histopathologic grade in endometrial cancer. Obstet Gynecol 1995;86:38-42.

16. Ben-Shachar I, Pavelka J, Cohn DE, et al. Surgical staging for patients presenting with grade I endometrial carcinoma. Obstet Gynecol 2005;105:487-93.

17. Corr BR, Carrubba A, Sheeder J, et al. Histopathology discrepancy of preoperative endometrial sampling and final specimen: how does this influence selective lymph node dissection? Int J Gynecol Cancer 2017;27:297-301.

18. Visser NCM, Reijnen C, Massuger L, et al. Accuracy of endometrial sampling in endometrial carcinoma: a systematic review and meta analysis. Obstet Gynecol 2017;130:803-13.

19. Ortoft G, Dueholm M, Mathiesen O, et al. Preoperative staging of endometrial cancer using TVS, MRI, and hysteroscopy. Acta Obstet Gynecol Scand 2013;92:536-45.

20. Cutillo G, Cignini P, Visca P, et al. Endometrial biopsy by means of the hysteroscopic resectoscope for the evaluation of tumor differentiation in endometrial cancer: a pilot study. Eur J Surg Oncol 2007;33:907-10.

21. Thanachaiviwat A, Thirapakawong C, Leelaphatanadit C, et al. Accuracy of preoperative curettage in determining tumor type and grade in endometrial cancer. J Med Assoc Thai 2011;94:766-71.

22. Kisielewski F, Gajewska ME, Marczewska MJ, et al. Comparison of endometrial biopsy and postoperative hysterectomy specimen findings in patients with atypical endometrial hyperplasia and endometrial cancer. Ginekologia Polska 2016;87:488-92.

23. Tanaka T, Terai Y, Ono YJ, et al. Preoperative MRI and intraoperative frozen section diagnosis of myometrial invasion in patients with endometrial cancer. Int J Gynecol Cancer 2015;25:879-83.

24. Göksedef BP, Akbayır O, Corbacıoğlu A, et al. Comparison of preoperative endometrial biopsy grade and final pathologic diagnosis in patients with endometrioid endometrial cancer. J Turk Ger Gynecol Assoc 2012;13:106-10.

25. Karateke A, Tug N, Cam C, et al. Discrepancy of preand postoperative grades of patients with endometrial carcinoma. Eur J Gynaecol Oncol 2011;32:283-5.

26. Su H, Huang L, Huang KG, et al. Accuracy of hysteroscopic biopsy, compared to dilation and curettage, as a predictor of final pathology in patients with endometrial cancer. Taiwan J Obstet Gynecol 2015;54:757-60.

27. Lago V, Martín B, Ballesteros E, et al. Tumor grade correlation between preoperative biopsy and final surgical specimen in endometrial cancer: the use of different diagnostic methods and analysis of associated factors. Int J Gynecol Cancer 2018;28:1258-63.

28. Garcia TS, Appel M, Rivero R, et al. Agreement between preoperative endometrial sampling and surgical specimen findings in endometrial carcinoma. Int J Gynecol Cancer 2017;27:473-8.

29. Okadome M, Saito T, Nishiyama N, et al. Prediction of histological types of endometrial cancer by endometrial cytology. J Obstet Gynaecol Res 2014;40:1931-9.

30. Batista TP, Cavalcanti CL, Tejo AA, et al. Accuracy of preoperative endometrial sampling diagnosis for predicting the final pathology grading in uterine endometrioid carcinoma. Eur J Surg Oncol 2016;42:1367-71.

31. Cho H, Kim YT, Kim JH. Accuracy of preoperative tests in clinical stage I endometrial cancer: the importance of lymphadenectomy. Acta Obstet Gynecol Scand 2010;89:175-81.

32. Talhouk A, Hoang LN, McConechy MK, et al. Molecular classification of endometrial carcinoma on diagnostic specimens is highly concordant with final hysterectomy: earlier prognostic information to guide treatment. Gynecol Oncol 2016;143:46-53.

33. Faber J, Lilian MF. How sample size influences research outcomes. Dental Press J Orthod 2014;19:27-9.

34. Landis JR, Koch GG. The measurement of observer agreement for categorical data. Biometrics 1977;33:159-74.

35. Nayak BK. Understanding the relevance of sample size calculation. Indian J Ophthalmol 2010;58:469-70. 
36. Benati M, Montagnana M, Danese E, et al. Aberrant Telomere Length in Circulating Cell-Free DNA as Possible Blood Biomarker with High Diagnostic Performance in Endometrial Cancer. Pathol Oncol Res 2020;26:2281-9.

37. Chiofalo B, Mazzon I, Di Angelo Antonio S, et al. Hysteroscopic evaluation of endometrial changes in breast cancer women with or without hormone therapies: results from a large multicenter cohort study. J Minim Invasive Gynecol 2020:27:832-9.

38. Helpman L, Kupets R, Covens A, et al. Assessment of endometrial sampling as a predictor of final surgical pathology in endometrial cancer. Br J Cancer 2014;110:609-15.

39. Di Spiezio Sardo A, De Angelis MC, Della Corte L, et al. Should endometrial biopsy under direct hysteroscopic visualization using the grasp technique become the new gold standard for the preoperative evaluation of the patient with endometrial cancer? Gynecol Oncol 2020;158:347-53.

40. Maneschi F, Ceccacci I, Perugini A, et al. Endometrial cancer: prognostic significance of risk classification based on pre-intraoperative findings. Arch Gynecol Obstet 2012;285:521-7.

41. Benedetti Panici P, Basile S, Maneschi F, et al. Systematic pelvic lymphadenectomy vs no lymphadenectomy in earlystage endometrial carcinoma: randomized clinical trial. J Natl Cancer Inst 2008;100:1707-16.

42. Bernardini MQ, May T, Khalifa MA, et al. Evaluation of two management strategies for preoperative grade 1 endometrial cancer. Obstet Gynecol 2009;114:7-15.

43. Neubauer NL, Havrilesky LJ, Calingaert B, et al. The role of lymphadenectomy in the management of preoperative grade 1 endometrial carcinoma. Gynecol Oncol 2009; 112:511-6.

44. Mariani A, Dowdy SC, Cliby WA, et al. Prospective assessment of lymphatic dissemination in endometrial cancer: a paradigm shift in surgical staging. Gynecol Oncol 2008;109:11-8.

45. Vitale SG, Capriglione S, Zito G, et al. Management of endometrial, ovarian and cervical cancer in the elderly: current approach to a challenging condition. Arch Gynecol Obstet 2019;299:299-315.

46. Vaknin Z, Ben-Ami I, Schneider D, et al. A comparison of perioperative morbidity, perioperative mortality, and disease-specific survival in elderly women ( $\geq 70$ years) versus younger women $(<70$ years $)$ with endometrioid endometrial cancer. Int J Gynecol Cancer 2009;19:879-83.

47. Vaknin Z, Perri T, Lau S, et al. Outcome and quality of life in a prospective cohort of the first 100 robotic surgeries for endometrial cancer, with focus on elderly patients. Int J Gynecol Cancer 2010;20:1367-73.

48. La Rosa VL, Garzon S, Gullo G, et al. Fertility preservation in women affected by gynaecological cancer: the importance of an integrated gynaecological and psychological approach. Ecancermedicalscience 2020;14:1035. Available online: https://ecancer.org/en/ journal/article/1035-fertility-preservation-in-womenaffected-by-gynaecological-cancer-the-importance-of-anintegrated-gynaecological-and-psychological-approach

49. Laganà AS, La Rosa VL, Rapisarda AM, et al. Comment on: "Needs and priorities of women with endometrial and cervical cancer". J Psychosom Obstet Gynaecol 2017;38:85-6.

50. Jančar N. Shranjevanje genetskega materiala pri onkoloških bolnicah = Fertility preservation in cancer patients. In Lukanovič A, Požun P, Lukanovič D (Eds.), Etični izzivi $\mathrm{v}$ ginekologiji, perinatologiji in reproduktivni medicini = Ethical challenges in gynaecology, perinatology and reproductive medicine. Available online: https://www.kclj. si/dokumenti/ETICNI_IZZIVI_V_GINEKOLOGIJI_ brosura_210x260_net.pdf

51. Del-Pozo-Lérida S, Salvador C, Martínez-Soler F, et al. Preservation of fertility in patients with cancer (Review). Oncol Rep 2019;41:2607-14.

52. Kim SY, Kim SK, Lee JR, et al. Toward precision medicine for preserving fertility in cancer patients: Existing and emerging fertility preservation options for women. J Gynecol Oncol 2016;27:e22.

53. Duska LR, Garrett A, Rueda BR, et al. Endometrial cancer in women 40 years old or younger. Gynecol Oncol 2001;83:388-93.

54. Vitale SG, Rossetti D, Tropea A, et al. Fertility sparing surgery for stage IA type I and G2 endometrial cancer in reproductive-aged patients: evidence-based approach and future perspectives. Updates Surg 2017;69:29-34.

55. Wang Q, Wang Q, Zhao L, et al. Endometrial cytology as a method to improve the accuracy of diagnosis of endometrial cancer: case report and meta-analysis. Front Oncol 2019;9:1-9.

56. Fujiwara H, Takahashi Y, Takano M, et al. Evaluation of endometrial cytology: cytohistological correlations in 1,441 cancer patients. Oncology 2015;88:86-94.

57. Fulciniti F, Yanoh K, Karakitsos P, et al. The Yokohama 
system for reporting directly sampled endometrial cytology: the quest to develop a standardized terminology. Diagnost Cytopathol 2018;46:400-12.

58. Casarin J, Bogani G, Serati M, et al. Presence of Glandular Cite this article as: Lukanovic D, Matjašič M, Kobal B. Accuracy of preoperative sampling diagnosis for predicting final pathology in patients with endometrial carcinoma: a review. Transl Cancer Res 2020;9(12):7785-7796. doi: 10.21037/tcr-20-2228

Cells at the Preoperative Cervical Cytology and Local Recurrence in Endometrial Cancer. Int J Gynecol Pathol 2020;39:522-8. 